\title{
Track-counting luminosity measurements in ATLAS
}

\author{
Patrawan Pasuwan*, on behalf of the ATLAS collaboration \\ Department of Physics, Stockholm University, Stockholm, Sweden \\ Oskar Klein Centre, Stockholm, Sweden \\ E-mail: prim.pasuwan@eern.ch
}

\begin{abstract}
At the LHC, the number of inelastic proton-proton collisions per second is proportional to the instantaneous luminosity. Track counting is one of the methods for luminosity measurement in the ATLAS experiment. It is done by counting the number of charged-particle tracks reconstructed in the inner detector using unbiased triggers, where the number of tracks scales with the number of interactions. Therefore, as long as the performance of track reconstruction and selection are independent of the luminosity and time, the average number of tracks per event can be used to measure the luminosity. A new track selection, which is less sensitive to changes in the inner detector conditions and shows a more stable performance over a large luminosity range, was introduced in 2017. Results from 2017 and 2018 data have shown good agreement between track counting and other luminosity algorithms, including LUCID which is the dedicated online and offline luminometer of the ATLAS detector.
\end{abstract}

7th Annual Conference on Large Hadron Collider Physics - LHCP2019

20-25 May, 2019

Puebla, Mexico

${ }^{*}$ Speaker. 


\section{Introduction}

The luminosity is an essential parameter for the physics programme of the ATLAS experiment [1]. The integrated luminosity is used when measuring a process cross-section $\sigma$ and when deriving the expected number of events $N_{\text {event }}$ originating from a certain process:

$$
\mathcal{L}_{\text {int }}=\frac{N_{\text {event }}}{\sigma}
$$

The total integrated luminosity of $156 \mathrm{fb}^{-1}$ was delivered to ATLAS by the LHC in LHC Run 2 (from 2015 to 2018) during stable beams of $p p$ collisions at $\sqrt{s}=13 \mathrm{TeV}$. ATLAS recorded $147 \mathrm{fb}^{-1}$ of the delivered luminosity. This is illustrated in Figure 1 [2]. Several detectors and algorithms are used in ATLAS to measure luminosity. The use of those luminometers, as they are called, allows for cross-checks of measurements and better control the systematic uncertainties [3].

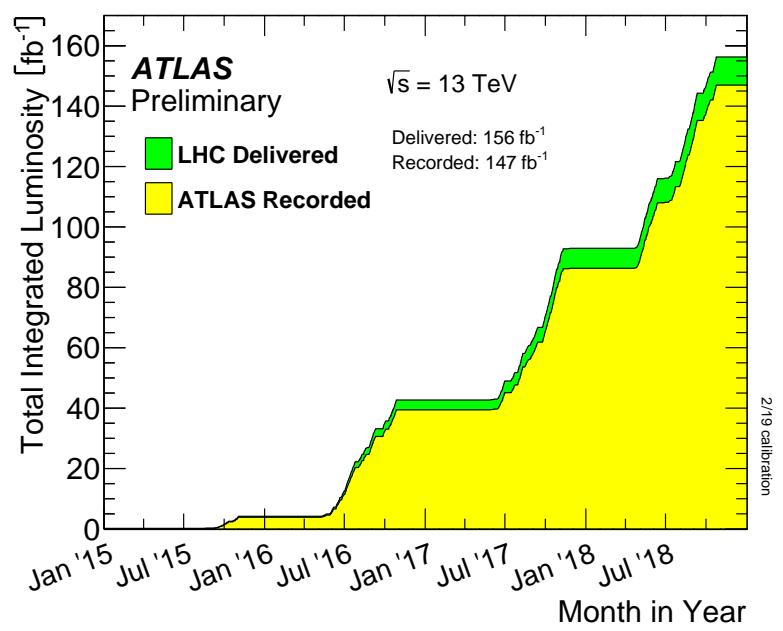

Figure 1: Cumulative luminosity that was delivered by the LHC (green) and recorded by ATLAS (yellow) in LHC Run 2 (from 2015 to 2018) during stable beams of $p p$ collisions at $\sqrt{s}=13 \mathrm{TeV}$ [2].

\section{Luminosity measurement}

The luminosity of a pair of proton bunches crossing each other, i.e. the per-bunch luminosity, can be obtained from:

$$
\mathcal{L}_{\mathrm{b}}=\frac{\mu f_{\mathrm{r}}}{\sigma_{\text {inel }}}
$$

where $\mu$ is the average number of inelastic $p p$ collisions per bunch crossing (i.e. pile-up parameter), $f_{\mathrm{r}}$ is the LHC revolution frequency of $11246 \mathrm{~Hz}$ [4], and $\sigma_{\text {inel }}$ is the $p p$ inelastic cross-section. Summing over the total number of colliding bunch pairs in the LHC ring $\left(n_{\mathrm{b}}\right)$, the total instantaneous luminosity can be obtained as:

$$
\mathcal{L}=\sum_{\mathrm{b}=1}^{n_{\mathrm{b}}} \mathcal{L}_{\mathrm{b}}=n_{\mathrm{b}}\left\langle\mathcal{L}_{\mathrm{b}}\right\rangle=n_{\mathrm{b}} \frac{\left\langle\mu_{\mathrm{vis}}\right\rangle f_{\mathrm{r}}}{\sigma_{\mathrm{vis}}},
$$


where $\left\langle\mathcal{L}_{\mathrm{b}}\right\rangle$ is the luminosity of colliding-bunch pair $\mathrm{b},\left\langle\mu_{\mathrm{vis}}\right\rangle$ is the visible (or effective) inelasticinteraction rate that the luminometer considered is sensitive to, and $\sigma_{\text {vis }}$ is the corresponding visible cross-section associated with that luminometer. The integrated luminosity can be obtained by integrating the instantaneous luminosity over time $(t)$ :

$$
\mathcal{L}_{\text {int }}=\int \mathcal{L} d t
$$

\section{Track-counting luminosity}

Track counting is one of the ATLAS luminosity algorithms. It is based on counting the number of reconstructed charged-particle tracks per event from the silicon detectors inside ATLAS's Inner Detector (ID) [5] using unbiased random triggers. These tracks are selected using the 2016 and 2017 track selections whose properties are listed in Table 1 [6]. Figure 2 shows the number of reconstructed tracks per event ( $N_{\text {tracks }}$ ) in simulated $Z \rightarrow \mu^{+} \mu^{-}+$pile-up events as a function of $\mu$ (from 0 to 100) for the two track selection criteria [6]. The linearity between $N_{\text {tracks }}$ and $\mu$ allows the luminosity to be deduced simply from $N_{\text {tracks. }}$. In practice, the absolute track-counting luminosity scale is determined by cross-calibrating with LUCID [7] in the quiescent period of a special LHC fill for van-der-Meer calibrations. LUCID is dedicated for luminosity measurement in the ATLAS detector. It is a set of photomultiplier tubes located 17 metres in either direction along the beam pipe. LUCID detects the Cherenkov light of particles from the $p p$ interaction.

\begin{tabular}{lcc}
\hline Track selection & $\mathbf{2 0 1 6}$ & $\mathbf{2 0 1 7}$ \\
\hline$p_{\mathrm{T}}[\mathrm{MeV}]$ & $>900$ & $>900$ \\
$|\eta|$ & $<2.5$ & $<1.0$ \\
$\left|d_{0} / \sigma_{d_{0}}\right|$ & $<7$ & $<7$ \\
\hline \multirow{2}{*}{ Number of silicon hits } & $\geq 9$ (for $|\eta| \leq 1.65)$ & $\geq 9$ \\
& $\geq 11$ (for $|\eta| \geq 1.65)$ & \\
\hline Number of pixel holes & 0 & $\leq 1$ \\
\hline \multicolumn{2}{c}{$\geq 1$ hit on one of the two innermost pixel layers } \\
\hline
\end{tabular}

Table 1: 2016 and 2017 track selection criteria [6]. The absolute value of the transverse impact parameter significance, $\left|d_{0} / \sigma_{d_{0}}\right|$, is defined as the absolute ratio of the transverse impact parameter with respect to the beam spot over the uncertainty in measuring $d_{0}$ [4]. A pixel hole is defined as a missing hit in an active pixel sensor.

The 2017 track selection has been changed with respect to the 2016 selection in order to reduce the dependence on ID running conditions. This results in a more stable track selection efficiency over the $\langle\mu\rangle$ range of 10 to 75 for both data and MC simulation as shown in Figure 3 [6]. The track selection efficiency is obtained from tracks corresponding to muons from $Z \rightarrow \mu^{+} \mu^{-}$events that pass the corresponding track selection. The 2016 track selection becomes less efficient as $\langle\mu\rangle$ increases, while the 2017 track selection efficiency is higher and varies less with $\langle\mu\rangle$. This demonstrates that the 2017 track selection clearly improves the performance of the track-counting luminosity measurement. 


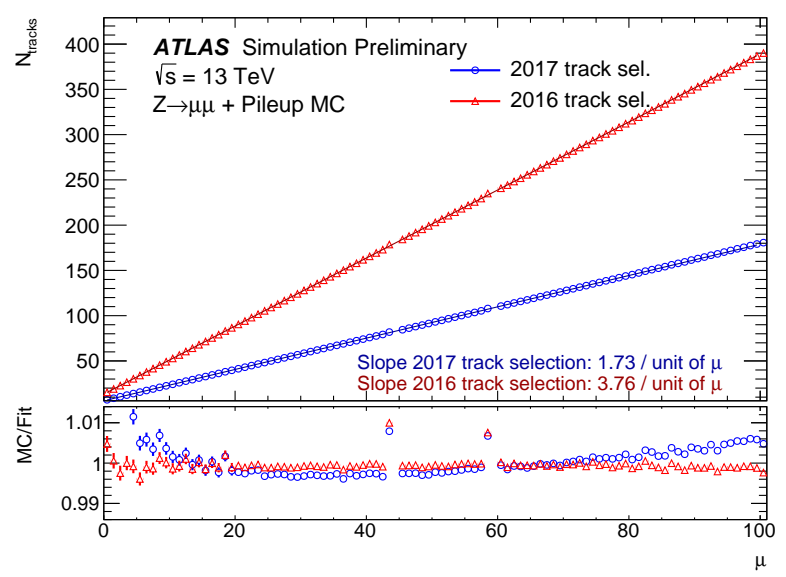

Figure 2: The number of reconstructed tracks per event in simulated $Z \rightarrow \mu^{+} \mu^{-}+$pile-up events as a function of $\mu$ for 2016 (red) and 2017 (blue) track selection criteria [6]. Each track selection is fitted with a first-order polynomial function, with the fitted slope indicated in the upper panel. The bottom panel shows the ratio between the simulated point and the fit value.

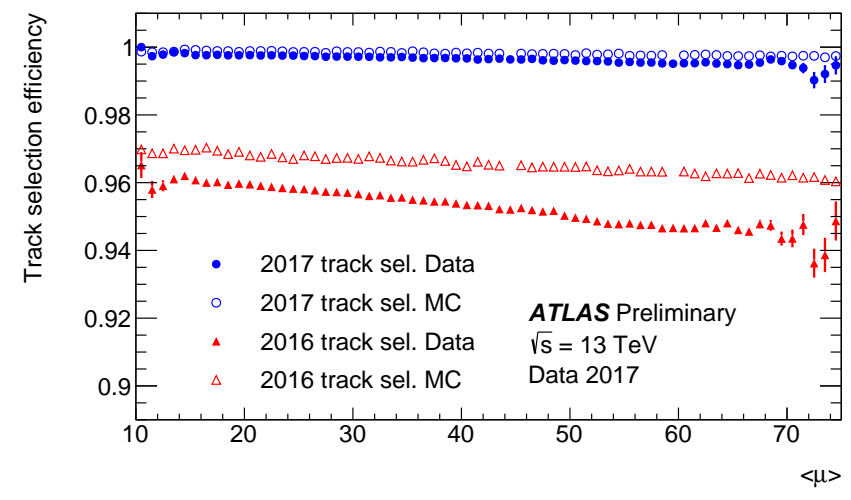

Figure 3: Track selection efficiency for muon tracks from $Z \rightarrow \mu^{+} \mu^{-}$events passing the 2016 (red triangles) or the 2017 (blue circles) track selection criteria as a function of $\langle\mu\rangle$ [6]. The results from data are presented in closed markers and from the MC simulation in open markers. The data were recorded in 2017.

A study of the LHC bunch structure dependence of track counting at moderate $\langle\mu\rangle$ values is performed with the 2017 track selection. The integrated luminosity ratio between track-counting and LUCID hit-counting algorithms is studied as a function of the bunch position in each LHC bunch train, using the data taken in 2017 with $\langle\mu\rangle=1$ and $\langle\mu\rangle=2$. The preferred LUCID algorithm for this study is called HitORBi. LHC fills 6019 and 6417 are selected, with properties shown in Table 2. Both fills have $25 \mathrm{~ns}$ spacing between each bunch pair. Fill 6417 has a repeated pattern of 8 filled bunch slots, followed by 4 empty bunch slots. This bunch pattern is referred to as $8 b 4 e$. During the fill, the LHC was operated at $\langle\mu\rangle=2$ for 4 hours and $\langle\mu\rangle=1$ for another 4 hours.

The results of this study are illustrated in Figure 4 [8]. The track/LUCID luminosity ratio for both $\langle\mu\rangle=1$ and $\langle\mu\rangle=2$ are not equal to unity. This could be caused by either the pile-up 
dependence, which is a well-established effect at higher $\langle\mu\rangle$ values [4], or a fill pattern dependence of the luminosity measurement. However, it is inconclusive which feature has a stronger effect on these particular low- $\langle\mu\rangle$ fills. With the possible exception of the first 8 bunch slots of fill 6019, there is no evidence, in either pattern, for a bunch-position dependence of the track/LUCID luminosity ratio beyond purely statistical fluctuations.

\begin{tabular}{ccccc}
\hline Fill number & $\langle\mu\rangle$ & Duration (hours) & Bunch pattern & Isolated bunches \\
\hline 6019 & 1 & 2 & 25 ns spacing & 3 \\
\hline \multirow{2}{*}{6417} & 1 & 4 & $8 \mathrm{~b} 4 \mathrm{e}$ & - \\
& 2 & 4 & & \\
\hline
\end{tabular}

Table 2: Summary of the LHC fills 6019 and 6417 [8].
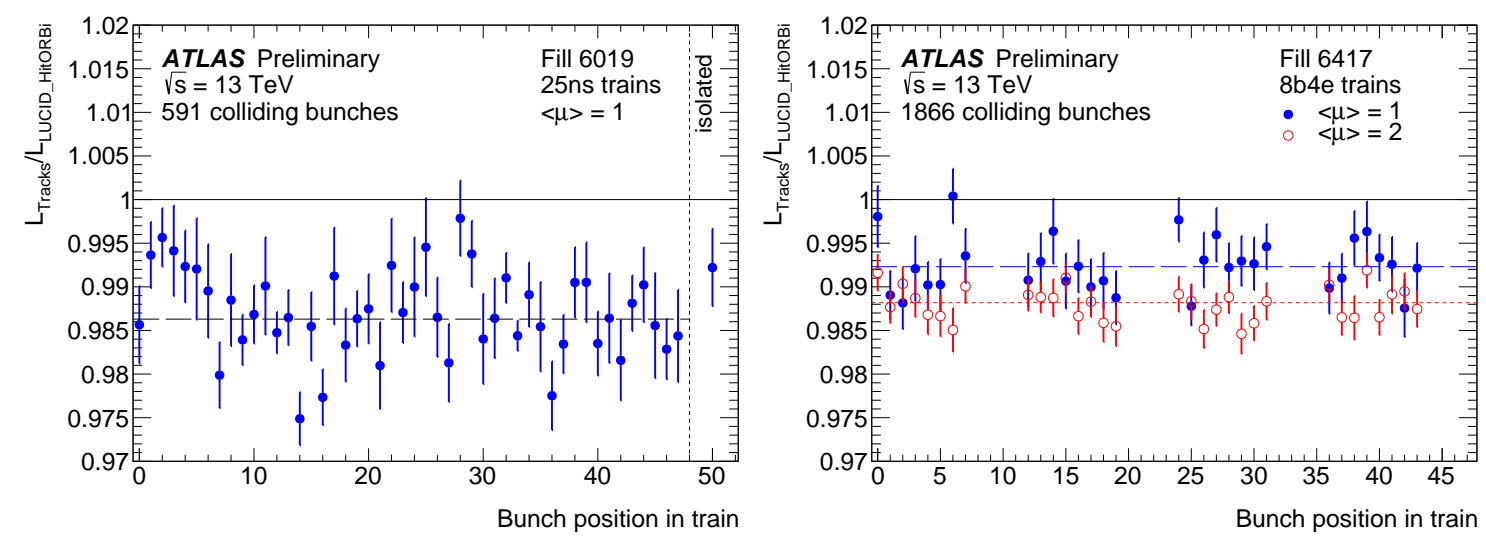

Figure 4: Ratio of the integrated luminosity measured by the track-counting and LUCID hitcounting algorithms during LHC fills 6019 (left) and 6417 (right), versus the position of the bunch within an LHC bunch train [8]. The closed blue (open red) markers represent $\langle\mu\rangle=1(\langle\mu\rangle=2)$. The horizontal dashed line represents the weighted average over bunch positions 0 to 47 . Bunch position zero indicates the first bunch in a train. The uncertainties are statistical only.

The luminosity values reported by various luminometers throughout the data-taking year are compared to each other in order to study the long-term stability. The results from the ATLAS runs recorded during 25 ns bunch-train running in 2017 [9] and 2018 [10] are shown in Figure 5. The run-integrated luminosity ratio between a given algorithm and LUCID (HitOR for 2017, C12 single-PMT for 2018) is presented as a function of day in the year. The luminosity of each algorithm is normalised to that of LUCID in the LHC fill marked by the red arrow.

A pile-up-dependent correction has been applied to LUCID luminosity recorded from the beginning of 2017 to August 12th, using the track-counting luminosity in LHC fill 6024 (July 29th) as a reference; another correction has been applied to LUCID for the later period, using the trackcounting luminosity in LHC fill 6259 (September 30th) as a reference. An upward correction of 2\% has also been applied to LUCID from June 5th to June 18th, based on comparisons with the trackcounting and calorimetry, TILE and EMEC luminosity algorithms [4]. For the data recorded in 

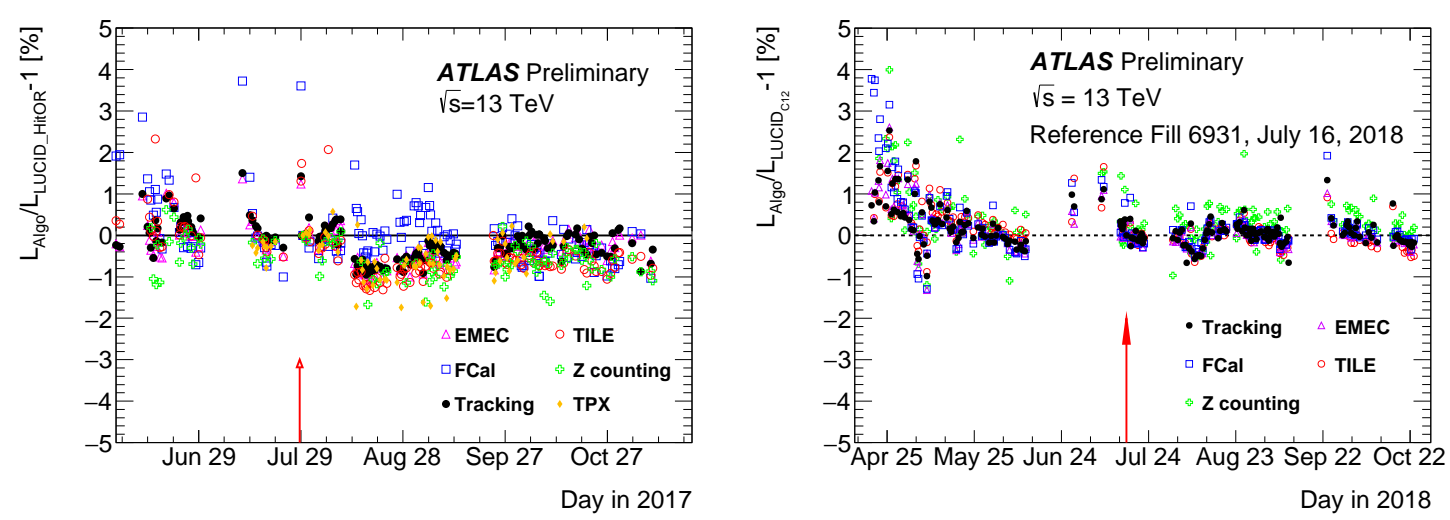

Figure 5: Fractional difference in the run-integrated luminosity between the LUCID preferred algorithm (left: HitOr for 2017, right: C12 single-PMT for 2018) and other luminosity algorithms. Each luminosity algorithm is normalised to LUCID in the data recorded in the LHC fill indicated by the red arrow [9].

2018, a pile-up-dependent correction is applied to the LUCID luminosity, using the track-counting luminosity in LHC fill 6931 (July 16th) as a reference.

The track-counting luminosity, using the 2017 track selection, shows good agreement with the luminosities measured by LUCID, EMEC, FCal, TILE, Z counting, and TPX throughout 2017 and 2018. More details on the respective algorithms can be found in Ref. [4].

\section{Summary}

Track counting is one of the luminosity algorithms used in ATLAS. It is based on counting the number of reconstructed charged-particle tracks per event from the silicon detectors using unbiased random triggers. Two track selection criteria have been defined, namely the 2016 and the 2017 track selections. The 2017 track selection has been changed with respect to the 2016 track selection in order to reduce the dependence on Inner Detector running conditions. With the 2017 track selection, the track-counting algorithm shows little luminosity, train position, and time dependence. The luminosity measured by track counting also shows good agreement with other luminometers.

\section{References}

[1] ATLAS collaboration, The ATLAS Experiment at the CERN Large Hadron Collider, JINST 3 (2008) S08003.

[2] ATLAS collaboration, Luminosity summary plots for 2018 pp data taking, https://twiki.cern.ch/twiki/bin/view/AtlasPublic/LuminosityPublicResultsRun2 .

[3] ATLAS collaboration, Luminosity determination in pp collisions at $\sqrt{s}=8$ TeV using the ATLAS detector at the LHC, Eur. Phys. J. C76 (2016) 653 [1608 . 03953 ].

[4] ATLAS collaboration, Luminosity determination in pp collisions at $\sqrt{s}=13$ TeV using the ATLAS detector at the LHC, ATLAS-CONF-2019-021, https://cds.cern.ch/record/2677054 . 
[5] ATLAS collaboration, Early Inner Detector Tracking Performance in the 2015 data at $\sqrt{s}=13 \mathrm{TeV}$, ATL-PHYS-PUB-2015-051, http://cdsweb.cern.ch/record/2110140 .

[6] ATLAS collaboration, Track-counting luminosity plots for the $2017 \sqrt{s}=13$ TeV high-luminosity running period, http://atlas.web.cern.ch/Atlas/GROUPS/PHYSICS/PLOTS/LUMI-2017-002 .

[7] G. Avoni et al., The new LUCID-2 detector for luminosity measurement and monitoring in ATLAS, JINST 13 (2018) P07017.

[8] ATLAS collaboration, Luminosity plots for the $2017 \sqrt{s}=13$ TeV low-luminosity runnning period, http://atlas.web.cern.ch/Atlas/GROUPS/PHYSICS/PLOTS/LUMI-2017-004 .

[9] ATLAS collaboration, Luminosity plots for the $2017 \sqrt{s}=13 \mathrm{TeV}$ high-luminosity running period, http://atlas.web.cern.ch/Atlas/GROUPS/PHYSICS/PLOTS/LUMI-2017-001 .

[10] ATLAS collaboration, Luminosity plots for the $2018 \sqrt{s}=13 \mathrm{TeV}$ high-luminosity running period, http://atlas.web.cern.ch/Atlas/GROUPS/PHYSICS/PLOTS/LUMI-2018-001 . 